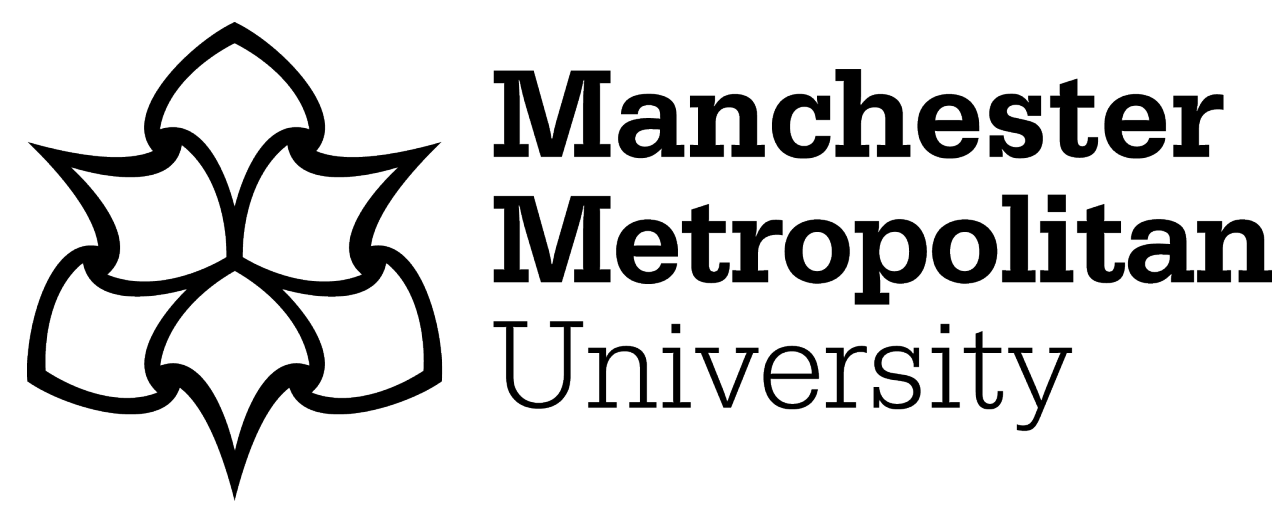

Khan, Wasiq, Hussain, Abir, Khan, Bilal, Nawaz, Raheel ORCID logoORCID: https://orcid.org/0000-0001-9588-0052 and Baker, Thar (2020) Novel Framework for Outdoor Mobility Assistance and Auditory Display for Visually Impaired People. In: 2019 12th International Conference on Developments in eSystems Engineering (DeSE), 07 October 2019 - 10 October 2019, Kazan, Russia.

Downloaded from: https://e-space.mmu.ac.uk/625616/

Publisher: IEEE

DOI: https://doi.org/10.1109/dese.2019.00183

Please cite the published version 


\section{Novel Framework for Outdoor Mobility Assistance and Auditory Display for Visually Impaired People}

\author{
Wasiq Khan \\ Department of Comouter Science \\ Liverpool John Moores University \\ Liverpoorl, United Kingdom \\ W.Khan@1jmu.ac.uk \\ Raheel Nawaz \\ School of Bussiness and Mangement \\ Science \\ Manchester Metropolitan University \\ Manchester, United Kingdom \\ R.Nawaz@mmu.ac.uk
}

\author{
Abir Hussain \\ Department of Comouter Science \\ Liverpool John Moores University \\ Liverpoorl, United Kingdom \\ A.Hussain@limu.ac.uk \\ Thar Baker \\ Department of Comouter Science \\ Liverpool John Moores University \\ Liverpoorl, United Kingdom \\ T.Baker@limu.ac.uk
}

\author{
Bilal Khan \\ Center for Environmental Implications \\ of Nanotechnology \\ University of California \\ Los Angeles, USA \\ M.Bilal@ucla.edu
}

\begin{abstract}
Outdoor mobility of Visually Impaired People (VIPs) has always been challenging due to the dynamically varying scenes and environmental states. Variety of systems have been introduced to assist VIPs' mobility that include sensor mounted canes and use of machine intelligence. However, these systems are not reliable when used to navigate the VIPs in dynamically changing environments. The associated challenges are the robust sensing and avoiding diverse types of obstacles, dynamically modelling the changing environmental states (e.g. moving objects, road-works), and effective communication to interpret the environmental states and hazards. In this paper, we propose an intelligent wearable auditory display framework that will process real-time video and multi-sensor data streams to: a) identify the type of obstacles, b) recognize the surrounding scene/objects and corresponding attributes (e.g. geometry, size, shape, distance from user), c) automatically generate the descriptive information about the recognized obstacle/objects and attributes, d) produce accurate, precise and reliable spatial information and corresponding instructions in audio-visual form to assist and navigate VIPs safely with or without the assistance of traditional means.
\end{abstract}

Keywords-Visual Impairment, Outdoor Mobility Assistance, Blind People Navigation, Object Recognition, Obstacle Detection, Healthcare Technology Framework, Data Fusion, Scene Recognition, Healthcare Aid

\section{INTRODUCTION}

Outdoor mobility has always been a challenge for the Visually Impaired People (VIP) due to numerous aspects specifically variety of moving objects, dynamic scenes and environmental conditions. World Health Organization [1] has reported 253 million VIPs (partially sighted or fully blind) worldwide, with 2 million within the UK ${ }^{1}$ and 30 million in the Europe ${ }^{2}$ and is ever increasing. Mobility of VIPs plays a fundamental role in enabling them to move around and walk safely in their neighbourhood. However, background scenes in outdoor environment change dynamically specifically outside a person's home. Lack of spatial information about live traffic, various obstacles and dynamics of moving objects on the route make safe mobility more difficult [2]. For example, a daily route taken by a VIP to the local supermarket may be familiar to them and they will have more confidence in navigating the route. However, if scaffolding or some development work appears along the route and reduces the public pavement, then their route has dynamically changed which may result in unexpected challenges.

Traditional ways to assist VIPs' outdoor mobility is to use trained guide dogs or rely on other people. The guide dogs may offer sufficient support but the associated costs for training them can approach up to $£ 36000$ per $\operatorname{dog}^{3}$ that is not a feasible option for many VIPs. In addition to traditional assistance, various technological solutions have been introduced such as sensors mounted canes and use of machine intelligence. However, these systems are not reliable when used to navigate the VIPs in dynamically changing outdoor environment. The most common challenges include detection and recognition of diverse types of obstacles/objects in live scenarios, dynamically modelling the changing outdoor environmental states (e.g. moving objects, road-works, weather conditions), and effective communication to verbally interpret the scenes/environmental states and different types of hazards.

In this paper, we propose a prototype autonomous auditory display framework that will process real-time videos and multi-sensor data streams to: a) Identify various obstacles/objects and corresponding spatial attributes (e.g. location, size, shape, distance from user), b) Generate the descriptive information about real-time scenes based on recognised obstacles and attributes, and c) produce accurate, precise and reliable information about spatial scenes and corresponding instructions in audio-visual form to assist and navigate VIPs safely with or without the assistance of traditional means.

One of the key challenge in this innovation will be to deal with real-time dynamics (e.g. light reflections, noisy data inputs, varying scenes and moving objects etc.) while processing the heterogeneous data streams to detect and recognise different obstacles/objects. We will develop efficient computer vision algorithms and construct appropriate intelligent models to overcome these challenges. 
The reminder of this paper is organised as follows. Section II addresses the existing systems related to VIP's mobility assistance. The proposed framework and its individual components are presented in Section III. Section IV presents the detailed experimental design followed by a conclusion in Section V.

\section{RELATED WORK}

The outdoor mobility is one of the major challenges VIPs face that seriously effects their social connections and quality of life. One of the major problem VIPs face during the outdoor mobility is the existence of obstacles/objects on pathways and dynamic surroundings. Recently, Royal National Institute of Blind People (RNIB) [1] reported that $90 \%$ of the VIPs have collided with an obstacle in local neighbourhood within a short period of three months. The most common obstacles reported are cars $(70 \%)$, bins of all kinds (64\%), street furniture (around 60\%), and advertising boards $(49 \%)$. In addition to temporary and fixed obstacles, safe road-crossings is also reported as one of the major problem for the mobility of VIPs. Over $67 \%$ participants reported the pedestrian crossings as unsafe for the mobility in local streets. Likewise, moving cyclists and new developments are reported as a "nightmare" for mobility in a dynamically changing environment. This indicates a drastic need for a technological solution that would fill the gap of local authorities, to improve the quality of VIPs' life in terms of safe and reliable mobility around neighbourhood.

Traditional ways to assist VIPs' outdoor mobility is to use trained guide dogs or rely on other people which holds expensive training costs. Alternative way of assistance is the use of long cane as a primary means of obstacle detection. Further improvements are made by using the electronic travel aids such as the laser cane and ultrasonic obstacle avoiders [3]. However, the sensing capabilities of these devices are not sufficient for VIP's reliable mobility or navigate from one point to another through unfamiliar outdoor environments. Recent state-of-the art technological advancement include Google glasses (https://x.company/) which introduced augmented reality based informative and attractive visual layer for the user. However, it works only for users with healthy vision as well as criticised and legal actions due to the concern of privacy issues. Likewise, the voice technology introduced in [4] uses the conversion of visual scene to sound using multiple cameras. However, it is limited to detect only few objects. Also, it works only in a pre-defined static environment and is unreliable in real-time varying scenes. ShopTalk [5], BlindShopping [6], and RoboCart [7] were introduced to locate a specific product in a supermarket by scanning barcodes on the shelves. Likewise data processing based methods presented in [8-12], however these approaches are limited to indoor environments and simulated scenarios only.

There exist a number of smartphone applications like BlindSquare $^{4}$, that support the navigation of VIPs between two points. It transforms some limited visual objects into spoken information, identifies the points of interest and street intersections dynamically using IOS built in navigation functionalities. The surrounding information is extracted with a $3^{\text {rd }}$ party application which is then transformed into speech messages and conveyed to traveller. Likewise, a prototype mobile application called ZebraX [13] that helps

\footnotetext{
${ }^{4}$ http://www.blindsquare.com/about/
}

VIPs towards and over a zebra crossing. Despite these technological advancements in relation to VIPs mobility, the reliability of all existing methodologies and technologies is questionable specifically, in terms of resolving the challenges associated with real time dynamically changing environmental scenes and VIPs' outdoor mobility.

In addition to VIPs' outdoor mobility, computer vision and data processing approaches have been applied into diverse application domains such as autonomous vehicles for the intelligent traffic sign recognition [14], video surveillance [15] for the real-time tracking object tracking, aerospace $[16,17]$ for live cockpit monitoring, underground mapping of objects [18], clustering of objects [19], biomedical $[20,21]$ for skin lesions characterisation and diabetes prediction, robotics [22-25] for multi-sensor data fusion, multi-obstacle detection [26] using laser scanner, self-driving cars [27], speech template matching [28, 29], healthcare technologies [30-35], and other adjacent domains. Likewise, recent research advancement include autonomous scene recognition using advance computer vision. For instance, [36] indicated that additional information captured through near-infrared within a conventional SLR camera improved the autonomous scene recognition significantly. Likewise, [37] presented a novel research on colour descriptors to recognise objects and scene. However, realtime scene recognition specifically in relation to dynamically changing outdoor environment is still a challenge.

For the first time, we are aiming to collect primary heterogeneous (multi-type sensors, images) dataset in relation to specific obstacles/objects and corresponding attributes (e.g. geometry, texture) that cause difficulty in VIPs' outdoor mobility. The proposed framework will use a composition of advanced computer vision and machine intelligence algorithms to process real-time streams captured through multi-type sensors and digital cameras to recognise various obstacles/objects and associated attributes within a fixed surrounding range. As the VIP will navigate, the realtime surrounding's information will be live streamed, processed, mapped to a pre-defined textual information and verbally interpreted to the VIP through headphones. The developed prototype will be portable, self-contained, and wearable with ability to assist VIPs for a reliable mobility and novelty of surrounding description with/without the assistance of secondary source.

\section{INTELLIGENT AUDITORY DISPLAY FRAMEWORK}

\section{A. Information Collection \& Dataset}

One of the main research element in the proposed framework is to collect discrete level information by questioning various VIP groups about challenges they face and obstacles types they mostly collide with, in routine outdoor mobility. Questionnaire will be designed to capture maximum information regarding VIPs outdoor mobility and consequences of hitting with obstacles/objects.

Following the information collected through surveys, a primary heterogeneous dataset will be collected for multiple obstacles/objects through various means that include a) multi-type sensors (e.g. Lidar, radar) to capture a novel dataset for various obstacles with diverse attributes, b) high quality image dataset for specific objects identified through questioning VIPs, c) existing data repositories. Main focus is to capture a novel heterogeneous dataset with corresponding features/attributes for each obstacle. 


\section{B. Data Processing}

This is the main technical research component of our proposed framework that focuses on processing of aforementioned dataset (images, heterogeneous sensor data) using advanced computer vision and signal processing approaches. Algorithm 1 will be developed to process multisensor data using signal/data processing approaches and time-frequency domain analysis (e.g. Wavelet/Fourier transforms). The original form of data will be transformed into a feature-space (e.g., width, height, geometric parameters, frequency, intensity, statistical parameters etc. for each obstacle) that will be used for the supervised model training.

Likewise, advanced computer vision algorithms will be developed to process the images dataset and extract the distinct attributes/features to be used for training supervised models (machine/deep learning). Specific challenges are to develop clever algorithms with ability to deal with real-time image/video data dynamics (e.g. image spatial registration, translations, rotation normalization etc.) and noisy inputs. For instance, light reflection, poor visibility, noisy sensor inputs, blurry images etc., are the most common type of issues.

The processed data in form of heterogeneous featurespace will be then further explored using variety of feature selections approaches to investigate the most distinctive/significant features that can represent variety of obstacles/objects optimistically to train effective machine models.

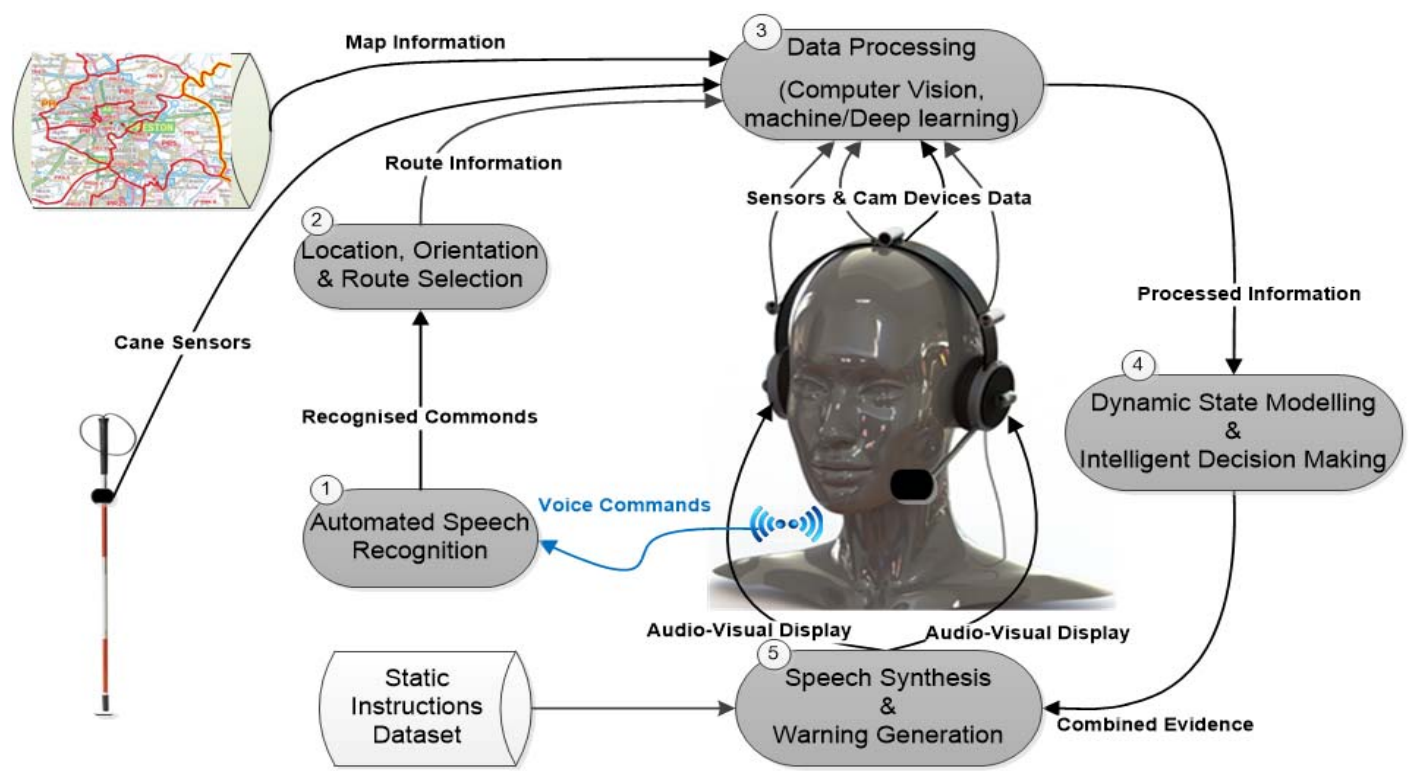

Fig. 1: Block diagram for the proposed wearable technology for VIPs assistance and mobility

Algorithm 1: Proposed Data Processing

Let $S$ to be a set of sensors producing data-streams,

Let $S_{1}=\{$ Lidar sensor data stream $\}$,

Let $S_{2}=\{$ Radar sensor stream $\}$,

Let $S_{3}=\{$ Thermal/Laser sensor stream $\}$.

$S=S_{1} \cup S_{2} \cup S_{3}$

Let $V$ to be a set of video data where

$V=\{v \mid v \in I, I$ is sequence of images $\}$.

Let $D \in R$ where $R$ is a real data and $D=S \cup V$

Let $F$ represents the set of features extracted from $D$ such that: $\forall D \exists f \in F$ where extracted features $F$ include \{colour, texture, geometry, histogram etc., etc.\}

C. Object Recognition and Obstacle Detection

Different machine/deep learning algorithms will be investigated while training on the processed data in form of optimistic feature-set. Specific focus will be to utilise convolutional neural networks (CNN) and multi-layer perceptron (MLP) to investigate the parametric configurations for the different machine learning models to get the optimum performance. Likewise, performance differences of machine learning algorithms when trained separately for sensor-based and image dataset, compared to be trained over a combined dataset will be investigated as illustrated in Algorithm 2.

Algorithm 2: Proposed Algorithm for data classification

Let $F$ represents a set of features determined as

Algorithm 1. Let Training $=\{t \in F \Rightarrow t \ltimes F\}$

Test $=\{t s \in F \Rightarrow t \ltimes F \& t \notin$ Training $\}$

For every selected Deep learning (DL) algorithm determined:

$E[$ Sensitivity] $=\{S: S \Rightarrow D L($ Training, Test $)\}$

$E[$ Specificity] $=\{S p: S p \Rightarrow D L($ Training, Test $)\}$

$E[F 1]=\{$ Sf1: Sf1 $\Rightarrow D L($ Training, Test $)\}$

$E[$ Precision] $=\{$ Spr: Spr $\Rightarrow D L($ Training, Test $)\}$

$E[$ Accuracy $]=\{$ Sa: $S a \Rightarrow D L($ Training, Test $)\}$ 


\section{Dynamic State Estimation}

A state estimation model will be developed to combine the evidences from multiple information sources and generate a belief score for the current state-space information (e.g. type of objects/obstacles \& corresponding attributes, location, distance from user etc.). The information sources include outcomes from classification and obstacle detection models, sensor measurements, and map databases etc. Theory of evidence and dynamic Bayesian networks are the potential approaches to be used for dynamic state estimation. Research will be carried out to develop and model the uncertain and dynamic situations specifically using a dynamic weight allocation to input information sources.

\section{E. Audio-Visual Scene Interpretation}

Fuzzy scores of live spatial states from above state estimator will be automatically mapped to a pre-defined static textual information dataset comprising spatial information such as objects' categories, colour names, obstacles shapes/geometry etc. The developed model will verbally interpret/transform the live spatial state information into an audio-visual explanation using speech synthesis. The verbal information and instructions will be produced to the user/VIP through the headphones.

The prototype framework (as shown in Figure 1) will be the integration of aforementioned components (Section III, $A-E)$ that will provide real time assistance to VIPs during outdoor mobility while live interpreting the surrounding scenes verbally through headphones.

\section{EXPERIMENTAL DESIGN}

To investigate the outlined research challenges, multiple experiments will be conducted using the heterogeneous dataset collected through multi-type sensors and camera images. For a fair evaluation of the data processing algorithms performance, various tests will be carried out to validate the proposed system using real-time and simulated scenarios. Dataset will be tuned and validated using standard approaches. Likewise, for the feature extraction, feature selection and dimensionality reduction, state-of-the-art methodologies such as principal component analysis, random forest, exhaustive feature selection etc., will be utilized to measure the attribute importance and effectiveness.

To validate the machine learning algorithms performance, standard cross-validation and leave-one-out strategies will be recursively used along with statistical measures from confusion matrix. The following experiments (labelled A to F) are designed with a consistent configuration parameters.

A) Once the data is processed and feature-space is extracted using time and frequency domain analysis, the attribute importance will be measured within entire featurespace using well-known Principal Component Analysis, Entropy/information gain, Pearson Correlation etc., to reduce the dimensions and data complexities.

B) Recurrent experiments of model train/test using exhaustive feature selection to identify the optimistic features/attributes representing variety of objects/obstacles.

C) The object classifier will be trained and tested on multi-type sensor based features using cross validation to measure the classification performance for sensor inputs.
D) The object classifier will be trained and tested on image based features using cross validation to measure the classification performance for camera inputs.

E) The object classifier will be trained and tested on combined features using cross validation to measure the performance of a hybrid classifier.

F) A series of experiments will be conducted to investigate the effects of real-time dynamics on system performance. For instance, simulated and real tests in the existence of light reflections, vibrations in camera devices, varying environmental conditions, moving background scenes etc.

For each experiment, various statistical metrics (i.e. sensitivity, specificity, accuracy, positive prediction rate, negative prediction rate, significance test such as $\mathrm{t}$-test/pvalue) as shown in Algorithm 2, will be used along with confusion matrices retrieved from deception classifiers to evaluate the performance of each specific case.

Once the proposed framework will be developed, two pilot studies/tests will be carried out. The integration of aforementioned components (Section III) will produce the proposed wearable platform. The main objective of the pilot tests will be to validate the ability of proposed technology to deal with real-time dynamics while producing a reliable mobility assistance and live scene interpretation to VIPs. Our experiments design will be used and a pilot test will be conducted to verify the $1^{\text {st }}$ prototype wearable platform in a controlled/lab and real environments in the presence of domain experts. Following the updates from $1^{\text {st }}$ pilot test, $2^{\text {nd }}$ Prototype test of proposed wearable platform will be conducted in the presence of experts from academia, industry, relevant organizations (e.g. Macular Society, RNIB, NHS) and VIPs.

\section{CONCLUSION}

Existing systems for VIPs' mobility assistance lacks the ability to deal with real-time dynamics such as varying background scene, moving objects, development works, noisy inputs etc. Traditional assistance is still used as a reliable source for VIPs' outdoor mobility. However, it is very expensive and cannot be afforded by most of the affected population even in developed countries. The proposed porotype framework is based on advanced machine intelligence approaches to deal with real-time dynamics using heterogeneous data processing and hybrid machine intelligence models. It will have the ability to recognise and interpret real-time scenes and spatial information (e.g. moving objects in background scene, obstacle type, location, and geometry) in audio-visual form to support VIPs' outdoor mobility with/without assistance. Furthermore, the proposed technological solution will be cost-effective with estimated value much less than traditional assistance. In future, we intend exploring the possibility of further incorporating textual data into the framework $[38,39]$.

\section{ACKNOWLEDGMENT}

The authors wish to thank Silent Taker Ltd. and Macular Society for their valuable discussions on the proposed framework.

\section{REFERENCES}

[1] R. Bourne et.al., "Magnitude, temporal trends, and projections of the global prevalence of blindness and distance and near vision 
impairment: a systematic review and meta-analysis," Lancet Glob Health. Vol. 5, No. 9, pp. e887-897, Sep. 2017, Online Available: http://www.who.int/mediacentre/factsheets/fs282/en/ [Accessed: May 2019].

[2] W. Wilson, "Who put that there! The barriers to blind and partially sighted people getting out and about," RNIB, Supporting People with Sight Loss, Feb, 2015, Onliane Available: https://www.rnib.org.uk/sites/default/files/Who\%20put\%20that\%20th ere $\% 21 \% 20$ Report $\% 20$ February\%202015.pdf [Accessed: March 2018].

[3] Y. Kumar et al., "RFID and GPS integrated navigation system for the visually impaired," $53^{\text {rd }}$ International Midwest Symposium on Circuits and Systems (MWSCAS), August 2010.

[4] D. Brown, T. Macpherson, and J. Ward, "Seeing with sound? exploring different characteristics of a visual-to-auditory sensory substitution device," Perception, vol. 40, no. 9, pp. 1120-1135, 2011.

[5] N. John, K. Vladimir, and C. Daniel, "ShopTalk: Independent blind shopping through verbal route directions and barcode scans," The Open Rehabilitation Journal, vol. 2, pp. 11-23, 2009.

[6] L. A. Diego, L. Tania, and L. P. Unai, "Indoor navigation and product recognition for blind people assisted shopping," Third International Workshop, IWAAL, IWANN, Torremolinos-Mlaga, Spain, June 810,2011

[7] G. Chaitanya, and K. Vladimir, "Robot-assited shopping for the blind: issues in spatial cognition and product selection," Intel Serv Robotics, vol. 01, pp. 237-251, 2008

[8] L. Betsworth, N. Rajput, S. Srivastava, and M. Jones, "Audvert: Using spatial audio to gain a sense of place," In Human-Computer Interaction- INTERACT 2013, Springer, pp. 455-462, 2013.

[9] J. Xiao, K. Ramdath, M. Iosilevish, D. Sigh, and A. Tsakas, "A low cost outdoor assistive navigation system for blind people," In Industrial Electronics and Applications (ICIEA), $8^{\text {th }}$ IEEE Conference on, Melbourne, Austrailia, July, 2013, pp. 828-833.

[10] V. Hoang, et al., "Obstacle detection and warning system for visually impaired people based on electrode matrix and mobile Kinect," Vietnam J Comput Sci, 2017, vol. 4, no. 02, pp. 71-83. DOI: https://doi.org/10.1007/s40595-016-0075-z.

[11] R. Jiang, Q. Lin, and S. Qu, "Let blind people see: real-time visual recognition with results converted to 3D audio", 2016, Online Available:

http://cs231n.stanford.edu/reports/2016/pdfs/218_Report.pdf [Accessed: Aug. 2018].

[12] K. Duarte, J. Cec'ilio, J. Silva, and P. Furtado, "Information and assisted navigation system for blind people," Proceedings of the $8^{\text {th }}$ International Conference on Sensing Technology, Liverpool, UK, Sep 2014.

[13] S. Mascetti, L. Picinali, A. Gerino, D. Ahmetovic, and C. Bernareggi, "Sonification of guidance data during road crossing for people with visual impairments or blindness," International Journal of HumanComputer Studies, Elsevier, vol. 85, pp. 16-26, January 2016.

[14] L. Chen, Q. Li, M. Li, and Q. Mao, "Traffic sign detection and recognition for intelligent vehicle," 2011 IEEE Intelligent Vehicles Symposium (IV), Baden-Baden, 2011, pp. 908-913. doi: 10.1109/IVS.2011.5940543

[15] J. S. Kim, D. H. Yeom, and Y. H. Joo, "Fast and robust algorithm of tracking multiple moving objects for intelligent video surveillance systems," in IEEE Transactions on Consumer Electronics, vol. 57, no. 3, pp. 1165-1170, August 2011. doi: 10.1109/TCE.2011.6018870.

[16] K. Wasiq, D. Ansell, K. Kuru, and M. bilal, "The flight guardian: autonomous flight safety improvement by monitoring aircraft cockpit instruments," Journal of Aerospace Information Systems, vol. 15, no. 04. pp. 203-214. ISSN 2327-3097, 2018, DOI: https://doi.org/10.2514/1.I010570.

[17] K. Wasiq, D. Ansell, K. Kuru, and M. Amina, "Automated aircraft instrument reading using real time video analysis," 2016 IEEE $8^{\text {th }}$ International Conference on Intelligent Systems (IS), Sofia, 2016, pp. 416-420. doi: 10.1109/IS.2016.7737454.

[18] M. Bilal, et al., "Inferring the most probable maps of underground utilities using Bayesian mapping model," Journal of Applied Geophysics, Elsevier, vol. 150, 2018, Pages 52-66, https://doi.org/10.1016/j.jappgeo.2018.01.006.

[19] K. Kuru K, and K. Wasiq, "Novel hybrid object-based non-parametric clustering approach for grouping similar objects in specific visual domains," Applied Soft Computing, Elsevier, vol. 62, pp. 667-701 2018, doi: 10.1016/j.asoc.2017.11.007.

[20] F. Riaz, S. Naeem, R. Nawaz, and M. Coimbra, "Active Contours Based Segmentation and Lesion Periphery Analysis for Characterization of Skin Lesions in Dermoscopy Images," IEEE Journal of Biomedical and Health Informatics, vol. 23, no. 2, pp. 489 500 ,

2019

1] M. Jahangir, et al., "An expert system for diabetes prediction using auto tuned multi-layer perceptron," Intelligent Systems Conference (IntelliSys), IEEE, 2017.

[22] R. C. Luo, M. Lin, and R. S. Scherp, "Dynamic multi-sensor data fusion system for intelligent robots," IEEE Journal on Robotics and Automation, vol. 04, no. 04, pp. 386-396, Aug. 1988. doi: $10.1109 / 56.802$

[23] K. Wasiq, and A. Badii, "Pathological gait classification and segmentation by processing the hip joints motion data to support mobile gait rehabilitation," Res Med Eng Sci. 2019, vol. 07, no. 03, doi: 10.31031/RMES.2019.07.000662.

[24] S. Ali et al., "An adaptive multi-robot therapy for improving joint attention and imitation of asd children," in IEEE Access, vol. 7, pp. 81808-81825, doi: 10.1109/ACCESS.2019.2923678

[25] T. Zaid, H. Ahmed, Y. Qureshi, and R. Nawaz, "Potentially guided bidirectionalized RRT for fast optimal path planning in cluttered environments," Robotics and Autonomous Systems, vol. 108, 2018 , pp. 13-27, doi: https://doi.org/10.1016/j.robot.2018.06.013.

[26] R. Labayrade, C. Royere, D. Gruyer, and D. Aubert, "Cooperative fusion for multi-obstacles detection with use of stereovision and laser scanner," Autonomous Robot, Springer, Sep. 2005, vol. 19, no. 02, pp. 117-140, doi:https://doi.org/10.1007/s10514-005-0611-7.

[27] H. Cho, Y. Seo, B. V. K. V. Kumar, and R. R. Rajkumar, "A multisensor fusion system for moving object detection and tracking in urban driving environments," IEEE International Conference on Robotics and Automation (ICRA), Hong Kong, 2014, pp. 1836-1843. doi: 10.1109/ICRA.2014.6907100.

[28] K. Wasiq, and K. Kuru, "An intelligent system for spoken term detection that uses belief combination," IEEE Intelligent Systems, vol. 32, no. 01, pp. 70-79, Feb. 2017. doi: 10.1109/MIS.2017.13.

[29] K. Wasiq, K. Crockett, and M. Bilal, "Adaptive framing based similarity measurement between time warped speech signals using Kalman filter," International Journal of Speech Technology, vol. 21 , no. 02, pp. 343-354, 2018, doi: https://dx.doi.org/10.1007/s10772018-9511-z.

[30] A. H. H. M. Mohamed, H. Tawfik, D. Al-Jumeily, and L. Norton, "MoHTAM: A technology acceptance model for mobile health applications," Paper presented at the Proceedings $-4^{\text {th }}$ International Conference on Developments in eSystems Engineering, DeSE 2011, doi:10.1109/DeSE.2011.79.

[31] A. J. Aljaaf, D. Al-Jumeily, A. Hussain, T. Dawson, P. Fergus, and M. Al-Jumaily, "Predicting the likelihood of heart failure with a multi level risk assessment using decision tree," Paper presented at 3rd International Conference on Technological Advances in Electrical, Electronics and Computer Engineering, TAEECE 2015, pp. 101-106, doi:10.1109/TAEECE.2015.7113608.

[32] M. Alloghani, A. J. Aljaaf, D. Al-Jumeily, A. Hussain, C. Mallucci, and J. Mustafina, "Data science to improve patient management system," Paper presented at the Proceedings - International Conference on Developments in eSystems Engineering, DeSE, 2018September 27-30, doi:10.1109/DeSE.2018.00012.

[33] A. J. Aljaaf, D. Al-Jumeily, T. K. Asman, A. J. Hussain, T. Baker, M. Alloghani, and J. Mustafina, "H-diary: Mobile application for headache diary and remote patient monitoring," Paper presented at the Proceedings - International Conference on Developments in eSystems Engineering, DeSE, , 2018-September 18-22. doi:10.1109/DeSE.2018.00010.

[34] A. J. Aljaaf, D. Al-Jumeily, H. M. Haglan, M. Alloghani, T. Baker, A J. Hussain, and J. Mustafina, "Early prediction of chronic kidney disease using machine learning supported by predictive analytics," Paper presented at the 2018 IEEE Congress on Evolutionary Computation, CEC 2018 - $\quad$ Proceedings, doi:10.1109/CEC.2018.8477876.

[35] A. J. Aljaaf, C. Mallucci, D. Al-Jumeily, A. Hussain, M. Alloghani, and J. Mustafina, "A study of data classification and selection 
techniques to diagnose headache patients," Applications of big data analytics: Trends, issues, and challenges, pp. 121-134, 2018, doi:10.1007/978-3-319-76472-6 6 .

[36] M. Brown and S. Süsstrunk, "Multi-spectral SIFT for scene category recognition," CVPR 2011, Colorado Springs, CO, USA, 2011, pp. 177-184. doi: 10.1109/CVPR.2011.5995637.

[37] K. van de Sande, T. Gevers, and C. Snoek, "Evaluating color descriptors for object and scene recognition," in IEEE Transactions on Pattern Analysis and Machine Intelligence, vol. 32, no. 9, pp 1582-1596, Sept. 2010. doi: 10.1109/TPAMI.2009.154.

[38] S. Ananiadou, T. Paul, and R. Nawaz, "Enhancing search: Events and their discourse context," International Conference on Intelligent Text Processing and Computational Linguistics. Springer, Berlin, Heidelberg, 2013.

[39] N. Batista et al., "Facilitating the analysis of discourse phenomena in an interoperable NLP platform," International Conference on Intelligent Text Processing and Computational Linguistics. Springer, Berlin, Heidelberg, 2013 